Erschienen in: Hyldgaard-Jensen, Karl/Zettersten, Arne (Hrsg.): Symposium on Lexicography III. Proceedings of the Third International Symposium on Lexicography May 14-16, 1986 at the University of Copenhagen. Tübingen: Niemeyer, 1988. S. 445-468. (Lexicographica: Series Maior 19)

DIETER HERBERG

ZUR PRAXIS DIACHRONISCHER MARKIERUNGEN IN ALLGEMEINEN EINSPRACHIGEN WÖRTERBÜCHERN

\title{
1: Zielstellung
}

Sowohl theoretisch als auch praktisch herrscht im Prinzip Konsens darüber, daß in einem brauchbaren allgemeinen einsprachigen wörterbuch die zur einzelnen lexikalischen Einheit gegebenen Informationen so vielseitig, so vollständig und so systematisch wie möglich sein sollten, denn der"gesamte Artikel ist eine Instruktion an den Wörterbuchbenutzer zum richtigen Verstehen und Gebrauch der behandelten lexikalischen Einheit" und für eine komplette Instruktion bedarf es außer der Definition "syntagmatischer und paradigmatischer, oft auch ikonographischer Information (Abbildungen) und einer Fülle weiterer Angaben und Markierungen" (HAUSMANN 1985, 374). In dieser Fülle weiterer Angaben und Markierungen nehmen diejenigen, die "pragmatische Informationen" vermitteln,d.h. solche Informationen,"die die Interpretation bzw. die produktion einer sprachlichen Äußerung über die grammatischen und semantischen Regeln hinaus - determinieren"(WIEGAND 1981, 144), einen besonderen Rang ein. 1 status, Funktion, systematik, Gewinnung und Vermittlung entsprechender Informationen, die unter Bezeichnungen wie "Konnotationen", "Wissen II", "nicht-denotative Informationen"zusammengefaßt werden, sind in der Lexikographie-Diskussion dieser Jahre immer wieder problematisiert worden. ${ }^{2}$ Dabei sind zwei Haupttypen von Informationen $\mathrm{zu}$ unterscheiden:

1 Andere Arten von zusätzlichen Angaben wären z.B. solche zur Aussprache oder zur Etymologie.

2 Um grundsätzliche Klärungen bemüht sich vor allem der Beitrag von WIEGAND (1981). Eine Zusammenschau der Problematik auf dem neuesten Forschungsstand bietet LUDWIG (1986), der auch die wichtige neue Literatur verzeichnet. 
(1) Informationen über Gebrauchspräferenzen und -restriktionen und

(2) Informationen, die emotionale Einstellungen des Sprechers zum benannten Gegenstand oder Sachverhalt anzeigen.

Diesen Informationen entspricht in der lexikographischen praxis ein Netz diasystematischer Markierungen, von dem freilich von Wörterbuch $\mathrm{zu}$ Wörterbuch in unterschiedlicher Breite und mit unterschiedlicher Konsequenz Gebrauch gemacht wird. Nach der jeweiligen Markierungsdimension sind vor allem diastratische, diachronische, diatopische, diatechnische, diafrequente und diaevaluative Markierungen anzutreffen. ${ }^{3}$

In diesem Beitrag soll speziell die d i a $c h r \circ n$ i $s \mathrm{ch} e$ Markierungsdimension unter die Lupe genommen werden, die - im Unterschied zu den meisten anderen - bisher kaum als Dimension sui generis, sondern vor allem im Zusammenhang mit Häufigkeitsangaben (SCHAEDER 1983), mit stilistischen Markierungen (ROSSIPAL 1973, SCHUMANN 1985) oder in bezug auf eine bestimmte Kommunikationsgemeinschaft, z.B. auf die der DDR (SCHMIDT 1982) behandelt worden ist, weshalb einige spezifische Aspekte diachronischer Markierungen in allgemeinen einsprachigen Wörterbüchern bisher nicht ausreichend zur Sprache gekommen sind. Unser Ziel ist es, einen Anstoß zu geben, auch die diachronischen Markierungen als einen belangvollen Typ diasystematischer Kennzeichnungen in die Diskussion um die möglichst zweckmäßige und benutzergerechte wörterbuchgestaltung einzubeziehen und ihren Status zu überdenken.

Zunächst wird die Markierungspraxis in den wichtigsten neueren deutschsprachigen, vergleichend auch in einigen älteren deutschen sowie in einigen anderssprachigen wörterbüchern charakterisiert (2.), danach sollen einige probleme diachronischer Markierungen relativ zu ihrem zweck diskutiert, ein paar Schlußfolgerungen gezogen und Empfehlungen für die praxis abgeleitet werden (3.), und schließlich fassen wir thesenhaft zusammen (4.).

3 Der Katalog von Markierungen, denen eigene Artikel im Internationalen Handbuch zur Lexikographie "Wörterbücher" gewidmet werden sollen, enthält außerdem noch die Markierung von Entlehnungen sowie normative Kommentare. 
2. Diachronische Markierungen in deutschsprachigen Wörterbüchern (mit einem Seitenblick aufs Ausland)

2.1. Wir haben unseren Erhebungen $15 \mathrm{zu}$ ihrer Zeit verbreitete synchronische Wörterbücher des Deutschen (mit dem Schwerpunkt deutsche Gegenwartssprache) zugrunde gelegt, die zwischen 1774 (ADELUNG) und 1985 (DUDEN-Bedeutungswörterbuch) erschienen sind (vgl. Ubersicht S. 46lf.).

Die Fragestellung war folgendermaßen eingegrenzt: Wird im gegebenen Wörterbuch die Markierungsdimension "Zeit"berücksichtigt? Wenn ja: Erfolgt die Markierung durch Prädikate aus einer Reihe von (definierten) Markierungsprädikaten oder durch offene pragmatische Kommentare? ${ }^{4}$ Wird mit Markierungsprädikaten gearbeitet: Aus welchen Markierungen konstituiert sich die Liste? Handelt es sich um statusgleiche Prädikate, und ist die Liste komplett oder defektiv? Die Fakten haben wir - soweit das möglich war den Einleitungen und ggf. den Abkürzungsverzeichnissen (A.) der Wörterbücher entnommen. ${ }^{5}$

2.2. Die Ubersicht(s:46lf.) zeigt,daß alle 15 wörterbücher diachronische Markierungen irgendwelcher Art vorsehen. Nur 7 Wörterbücher allerdings geben in ihren Einleitungen - wenn auch oft sehr knappe - Erläuterungen $z u$ den verwendeten Markierungsprädikaten, während diese in den übrigen wörterbüchern lediglich in den Verzeichnissen der Abkürzungen bzw. der Symbole auftauchen.

2.3. Es lassen sich drei Verfahren diachronischer Markierung feststellen:

- Markierungen mittels einer Reihe von prädikaten wie in (5), (7), (8), (11), (12), (13), (14), (15), mitunter auch mit nur einem Prädikat (veraltet in (3), (4) und (10);

- Markierung mittels einer Gruppe von Symbolen wie in (2) oder unter Verwendung eines Symbols wie in (6) und (9);

- Markierung mittels offener pragmatischer Kommentare.

4 Zum Problem geschlossener und offener pragmatischer Markierung vgl. WIEGAND (1981, $177 \mathrm{ff.})$.

5 Die Überprüfung der Handhabung der Markierung im Wörterverzeichnis des jeweiligen wörterbuches auf ihre Konsequenz hin j.st dagegen nicht Anliegen dieses Beitrages. 
Das letztgenannte Verfahren läßt sich mit der hier verwendeten Untersuchungsmethode nur unzulänglich ermitteln. Während es bei ADELUNG ausgiebig und bei einigen neueren wörterbüchern zusätzlich ebenfalls angewandt wird ${ }^{6}$, gehen die Einleitungen darauf nicht ein, so daß nur eine Durchsicht des gesamten wörterverzeichnisses zur vollständigen Erfassung der im einzelnen ggf. gewählten Formen führen könnte. Solche Kommentierungen lassen wir daher im folgenden außer acht.

2.4. Auf den ersten Blick wird sichtbar, daß das Inventar der vorgefundenen Markierungsprädikate trotz der gemeinsamen Beziehung zur Dimension "Zeit" recht heterogenen Charakters ist. Der größte Teil der Prädikate bezieht sich auf Lexeme, Sememe oder Verwendungen und ist also $s \mathrm{p} \mathrm{a} c \mathrm{~h}$ bezogen. Dazu zählen wir ursprünglich, veraltet, veraltend, nationalsozialistisch/ nazistisch, neu, neugebildet, Neuwort, Neuprägung, Neubedeutung, Modewort, Kunstwort.

Ein kleinerer Teil von Markierungen bezieht sich dagegen ausdrücklich auf die Gegenstände oder Sachverhalte vergangener Zeiten und ist damit $\mathrm{s}$ a $\mathrm{c}$ h bezogen: historisch und früher. 7 Obwohl sie in den Einleitungen unter den diachronischen Markierungen aufgeführt werden, sind sie mitnichten als solche $z u$ verstehen, denn selbstverständlich kann und muß bei Bedarf mit den so gekennzeichneten Ausdrücken auch gegenwärtig auf die entsprechenden Gegenstände oder Sachverhalte der Vergangenheit Bezug genommen werden. Diese sachbezogenen Kennzeichnungen interessieren uns im gegebenen Zusammenhang nicht und bleiben im weiteren außerhalb der Betrachtung.

2.5. Die Liste der $s$ p $r$ a $c$ h bezogenen Markierungsprädikate umfaßt ihrerseits sehr verschiedenartige Kommentarausdrücke, wie ein flüchtiger Blick lehrt. Darauf kommen wir zurück, wollen

6 Im HDG z.B.: heute noch schweiz. (Füselier).

7 Die Markierung altertüme Ind (WDG), die als "Stilfärbung" verstanden wird, haben wir hier bewußt unberücksichtigt gelassen. In diesem Sinne ist wohl auch die nicht definierte Markierung altertümlich in PEKRUN zu interpretieren. 
an dieser Stelle aber bereits eine andere Auffälligkeit registrieren: Sämtliche 15 wörterbücher kennzeichnen in irgendeiner Form die lexikalische $A \mathrm{r} c \mathrm{~h}$ a $i \mathrm{~s} i \mathrm{e} \mathrm{r}$ u $\mathrm{g}$, aber nur drei nehmen auf die $\mathrm{N}$ e $\circ \mathrm{l} \circ \mathrm{g} i \mathrm{e}$ Bezug. Sieht man einmal von der etwas aus dem Rahmen fallenden Markierung Kunstwort ("neuzeitliche gelehrte oder gewerbliche wortbildung") im Sprach-Brockhaus $a b$, so sind es nur zwei der großen deutschsprachigen Wörterbücher (das "Wörterbuch der Deutschen Sprache" von J. H. CAMPE und - mehr als 150 Jahre danach - das "Wörterbuch der deutschen Gegenwartssprache", hrsg. von R. KLAPPENBACH und W. STEINITZ), die sich der Mühe unterzogen haben, auch die lexikalischen Innovationen durch Markierungen auszuzeichnen. Dieser bemerkenswerte widerspruch insbesondere war es, der uns zu einigen Uberlegungen über die Funktion, den Status und die spezifische Problematik diachronischer Markierungen in synchronischen Wörterbüchern angeregt hat (vgl. 3).

2.6. Zuvor soll ein flüchtiger Blick auf einige ausländische Wörterbücher desselben Typs geworfen werden, um einen Eindruck von der dort geübten Praxis in bezug auf die Markierungsdimension "Zeit" zu gewinnen (vgl. Ubersicht S.464). Dieser in keiner Weise repräsentative Einblick in einige russische, tschechische, französische und englische wörterbücher zeigt in vielerlei Hinsicht Parallelen zur praxis in deutschsprachigen wörterbüchern, so in bezug auf die nicht immer deutliche Trennung von sachbezogenen und sprachbezogenen Markierungen und - innerhalb der letzteren - der Markierungsdimensionen"Zeit"und "Stil". Unser besonders Interesse gilt natürlich der Beobachtung, daß auch in den einsprachigen wörterbüchern anderer Sprachen der Pol "Alt" immer, der Pol "Neu" dagegen nur bei einem Teil der Wörterbücher mit Markierungsprädikaten versehen wird. Immerhin ist festzustellen, daß "das beste einbändige wörterbuch der Welt" (HAUSMANN 1983, 122), der einbändige Petit Robert, in bezug auf die Dimension "Zeit" die vollständige, die Pole "Alt" und "Neu" umfassende Markierungsskala benutzt. Ein auffälliger Unterschied $z u$ den deutschsprachigen Wörterbüchern ist, daß im 
Falle der Kennzeichnung lexikalischer Innovationen nicht ein Bündel unterschiedlicher Markierungen wie bei CAMPE und WDG, sondern jeweils nur ein einzelnes Prädikat (novoe oder néologisme oder modern use) verwendet wird.

Das diese kurze Bestandsaufnahme abschließende Fazit hat festzuhalten, daß das System der diachronischen Markierungen der meisten deutschen, aber auch vieler auslänłischer Wörterbücher in der Weise defektiv ist, daß der regelmäßigen Markierung auf der Seite der Archaisierung ein Markierungsdefizit auf der Seite der Neologie gegenübersteht. ${ }^{8}$

3. Was sind und was sollen diachronische Markierungen in allgemeinen einsprachigen Wörterbüchern?

3.1. Bevor wir möglichen Ursachen der offensichtlich disproportionierten diachronischen Markierungen erörtern, legen wir uns zunächst die Frage vor: Worum handelt es sich eigentlich bei der Kennzeichnung von lexikalischen Einheiten in bezug auf die Dimension "Zeit"? Daß die Auffassungen davon nicht übereinstimmen, verraten bereits die zusammenfassenden Bezeichnungen für diese Art von Markierungen. Es kommen verschiedene Aspekte ins Spiel, je nachdem ob etwa die Rede ist von der "Kennzeichnung der zeitlichen Zuordnung" (WDG), von "Angaben zur zeitlichen Verbreitung" (Brockhaus-Wahrig), von "Markierungen, die Hinweise auf zeitlich begrenzten Gebrauch des Lexems geben" (LUDWIG), von der "historischen Perspektive" (ŠMELEVA), von der

8 In Analogie zu einer Frage, die WIEGAND $(1981,179)$ in bezug auf eine ähnlich auffällige Einseitigkeit bei der Kennzeichnung evaluativer Lemmata und Redewendungen - nämlich nur mit dem Prädikat abwertend - gestellt hat, könnte man angesichts unserer Bilanz fragen: Sind die Gegenwartssprachen und ist insbesondere die deutsche Gegenwartssprache etwa nur dadurch gekennzeichnet, daß ihr Wortschatz infolge von Alterungserscheinungen langsam dahinschwindet? 
"Dimension der Aktualität" (ROSSIPAL) oder vom "Alter der wörter" (HAUSMANN 1977); mit der im HDG verwendeten Formulierung "Die zeitliche Kennzeichnung" wird die Sache am neutralsten benannt.

Es ist relativ leicht herauszufinden, daß die Kategorisierungen, die jeweils eine bestimmte seite des problems akzentuieren, nicht generell und auch nicht auf alle einzelnen Markierungsprädikate anwendbar sind. Das ist wohl Ausdruck der besonderen Problemlage, die sich - im Unterschied etwa zu Markierungsdimensionen wie "areale Verteilung" oder "fachsprachlicher Gebrauch" - für die Markierungsdimension "Zeit" bei einer synchronischen Wortschatzdarstellung zwangsläufig ergeben muß. Gilt es doch, Dynamisches im Statischen, Elastisches im Stabilen, Sich-Wandelndes im Bestehenden oder - bildlich gesprochen den Film in der Momentaufnahme sichtbar zu machen. Diese Verpflichtung für die Lexikographie der Gegenwartssprache ergibt sich daraus, daß "reine"Synchronie eine Fiktion ist, denn "mindestens vier Generationen sprechen z.B. das gegenwärtige Deutsch" (WIEGAND/KUČERA 1981, 146), d.h. die "Großeltern gebrauchen wörter, die der Enkel entweder nicht mehr versteht oder zumindest als veraltet ablehnt. Der Enkel wiederum nimmt wörter in den Mund, die den Großeltern barbarisch, modernistisch oder ausländisch vorkommen" (HAUSMANN 1977, 113).Diese in der synchronie steckende. Diachronie ist es, die der Lexikograph von seinem historischen standort aus, ausgerüstet mit seiner Sprachkompetenz und - hoffentlich - mit einer aussagekräftigen Kartothek, als Angehöriger seiner Kommunikationsgemeinschaft sowohl bei der Auswahl des Wortschatzes als auch bei der diachronischen Markierung einzelner Wortschatzelemente einzufangen bemüht sein muß. Läßt man jeweils schwankungen, die sich aus der im jeweiligen wörterbuch verfolgten Konzeption und aus dem zugrunde gelegten Erfassungszeitraum ergeben können, einmal beiseite, so besteht der Hauptteil oder das Zentrum des in Wörterbüchern des hier behandelten Typs versammelten wortschatzes unter dem zeitlichen Aspekt aus der $g$ e $n$ e $r$ a $i \circ n$ s $n s p$ e $z$ if i s che n, d i c hron is ch merkmallos e $n$ 
452

Lexik. "Und mit Recht bleiben diese relativ zur Dimension "Zeit" neutralen lexikalischen Einheiten, die im gesamten Erfassungszeitraum, also von seinem Beginn bis zum Zeitpunkt der Bearbeitung des Wörterbuches, ohne Einschränkungen von allen verwendet werden konnten, ohne diachronische Markierung. Diachronisch markiert werden muß dagegen alles, was am Beginn des Erfassungszeitraumes noch nicht, jedoch an seinem Ende im allgemein verbreiteten Wortschatz vorhanden ist ebenso wie das, was zum Bearbeitungszeitpunkt $\mathrm{n}$ i $\mathrm{ch} t \mathrm{~m}$ e $\mathrm{h} r$ ohne Einschränkung verwendet werden kann, aber noch vorhanden ist. Dabei handelt es sich u.E. in jedem Fall um "Diachronie nach rückwärts" und nicht im Falle never Wörter um "Diachronie nach vorwärts", wie HAUSMANN (1977, $112 \mathrm{ff.})$ meint. Unterschiedlich ist allein der Betrachtungswinkel: Einmal interessieren Tatsache und Zeitpunkt des Aufkommens einer in den Wortschatz integrierten Einheit (Neologie), die im anderen Fall uninteressant sind (Archaisierung); auf der anderen Seite (Archaisierung) werden aus dem Alterungsprozeß sich ergebende Verwendungsbeschränkungen signalisiert, wohingegen Gebrauchsrestriktionen auf der Seite der Neologie weniger relevant $z u$ sein scheinen. Dieser in der Natur des Sprachwandels liegende unterschiedliche Blickwinkel auf die Pole der diachronischen Skala ist wohl in erster Linie für die festgestellte Einseitigkeit bei der diachronischen Markierung verantwortlich. Anscheinend haben die sekundär mit der diachronischen Markierung der Alterung gegebenen Bezüge zur stilistischen "Höhenlage" (vgl. z.B. ROSSIPAL 1973, 52 ff. und SCHUMANN 1985, die solche Umdeutungen und Beziehungen belegen) oder zur Frequenz (vgl. SCHAEDER 1983) die Lexikographen in stärkerem Maße zur Markierung dieses Poles motiviert als das in bezug auf die unter diesen Aspekten weniger eindeutig und ergiebig erscheinenden Innovationen der Fall ist. Daß deren Markierung auch unter dem Verwendungsaspekt ebenfalb Bedeutung hat, wurde schon

9 Unter dem Aspekt anderer Markierungsdimensionen sind z.B. der der nichtfachgebundenen Kommunikation dienende, der gruppenunspezifische oder der iberrenional verwendete Teil der Lexik zentrale, unmarkierte Teile des Nortschatzes. 
von ROSSIPAL (1973, 53) diskutiert; nur geht es hier weniger um signalisierte Restriktionen,sondern viel eher um mögliche Präferenzen des Gebrauchs, denn im kommunikationsgemeinschaftlichen Durchschnitt verleiht nach ROSSIPAL (1973) der "Faktor /+aktuell/...dem Wort...einen positiven Wert" und das "Merkmal /+aktuell/ bekommt daher auch leicht eine gruppensprachliche Funktion (man ist "in" oder "out")" (S.53). 10

Und SMELEVA (1975/1982), der die diachronische Markierung "in Wörterbüchern der Hochsprache als ein Aspekt ihrer stilistischen Beschreibung" gilt, der "unumgänglich und notwendig" ist, meint, "eine stilistische Ausrichtung würde wohl in den wörterbüchern auch die Bewertung "neu" erhalten, wenn die Möglichkeit bestünde, sie konsequent und genau anzuwenden, und wenn sie, als Folge davon, sich in der Wörterbuchpraxis behaupten könnte" (207).

3.2. Wir wollen hier dafür plädieren, daß nicht diese selbstverständlich - und zwar sowohl bei archaisiertem als auch bei innovativem Wortgut - bestehenden Bezüge und kommunikativ wichtigen Informationen, die durch diachronische Markierungen über Gebrauchsrestriktionen bzw. -präferenzen oder Frequenz mitvermittelt werden, zum allein leitenden Gesichtspunkt in der Praxis diachronischer Markierung gemacht werden, sondern daß die Markierungsdimension "Zeit" auch als Dimension eigenen Werts und Rechts gesehen wird. Sie hat - vollständig und nicht defektiv praktiziert - nach unserer Meinung dem Wörterbuchbenutzer (gleich, ob er ein sprachliche Suchfragen stellender, ein sprachwissenschaftlich motivierter oder ein fremdsprachenlernender Benutzer ist)wesentliche Kenntnisse und Einsichten zu vermitteln in die komplexe Struktur des Gegenwartswortschatzes, die er so nirgend sonst geboten bekommt. Wenn "Nutzen und Wert solcher Wörterbücher (..) im Bereich der exhaustiven Wortschatz-

10 Nur angedeutet soll werden, daß in Abhängigkeit von der Kommunikationssituation aber auch von subjektiven Faktoren des Sprachbenutzers in bestimmten Fällen genau umgekehrt die $\mathrm{B}$ e $\mathrm{v} \circ \mathrm{r} \mathbf{z} \mathrm{u} \mathrm{g}$ u $\mathrm{n} g$ veralteten Wortgutes bzw. die $\mathrm{V}$ e $\mathrm{r}$ $\mathrm{m}$ e $\mathrm{i} d \mathrm{u} \mathrm{n} \mathrm{g}$ von Neologismen als opportun erscheinen kann. 
454

dokumentation und spezialisierten Wortschatzexplikation" liegen, wie KüHN:/PUSCHEL $(1982,132)$ nicht zu Unrecht meinen, so ist dem auch durch möglichst konsequente Verwendung diasystematischer Markierungen Rechnung zu tragen. Bezogen auf diachronische Markierungen heißt das aber:

Die Kennzeichnung von Mobilität und Dynamik im Gegenwartswortschatz muß Vergehen $u n d$ Werden, Ausscheiden $u n d$ Aufkommen, Abgänge u $\mathrm{n}$ d Zugänge umgreifen und darf sich keiner aus pragmatischen Erwägungen erwachsenden Einseitigkeit schuldig machen. Nur so kann sich das wörterbuch "als ein lebendiges Gebilde, als Spiegelbild einer sich rasch verändernden Wirklichkeit" erweisen, "indem es nämlich dem Benutzer die Geschichtlichkeit der Erscheinungen und Abläufe bewußtmacht" (MATTAUSCH 1979, 215). "Spürsinn und die Fähigkeit, Entwicklungstendenzen rechtzeitig $z$ u erfassen, werden von den Autoren eines solchen Werkes in besonderem Maß gefordert, soll der Gegenstand nicht zu einem künstlich konservierten, lebensabgewandten Gebilde erstarren" (ebenda).

3.3. Auch wenn das WDG mit seiner Pionierleistung der differenzierten Markierung von Neologismen bisher in der deutschsprachigen Lexikographie keine Nachfolge gefunden hat, so bedeutet das natürlich nicht, daß andere wörterbücher keine Neologismen aufgenommen hätten. So heißt es z.B. in bezug auf das HDG, das die Markierungspraxis des WDG hinsichtlich der Neologismen bedauerlicherweise nicht fortgeführt hat: "Das Handwörterbuch ... ergänzt in angemessenem Umfang den Stichwortkatalog durch Neologismen, die sich seit den siebziger Jahren ergeben haben (Industrieroboter, Mikroprozessor, Chip, Digitaluhr, um nur einige zu nennen)." (KЕMPCKE 1985, 133)

Und der in den wörterbucheinleitungen des DUDEN-GWB und des DUDEN-DUW zu findende Satz: "Neuwörter und Neubedeutungen sowie Modewörter sind nicht besonders gekennzeichnet" verrät nicht nur daß solcherart Phänomene im wörterbuch enthalten sind, sondern zeigt doch wohl auch, daß eine Benutzererwartung nach "besonderer Kennzeichnung" der Neologismen unterstellt und als legitim anerkannt, aber eben im gegebenen Fall nicht erfüllt wird. Wa- 
rum nicht? Soll man aus einer solchen Nichtmarkierungspraxis auf eine andere als z.B. die im WDG zugrunde gelegte Neologismenauffassung schließen? Die Wörterbucheinleitungen lassen den Benutzer darüber im unklaren (vgl. in bezug auf die HDG-Einleitung HERBERG 1986).

Zweifellos hat sich die Unterentwicklung der deutschen Neologismenlexikographie (Neographie), d.h. das Fehlen eines oder mehrerer größerer Neologismenwörterbücher ${ }^{11}$ nachteilig auf die Beschäftigung mit theoretischen problemen der Neologie ausgewirkt, während umgekehrt die Defizite in der linguistischen Aufarbeitung mitverantwortlich für die Lücken in der angemessenen lexikographischen Darstellung von Neologismen in allgemeinen einsprachigen Wörterbüchern des Deutschen sein dürften. Die Enthaltsamkeit bei der Markierung von Neologismen in allgemeinen Wörterbüchern des Deutschen erscheint in Anbetracht des Fehlens eines eigenständigen Neologismenwörterbuches - im Unterschied etwa zum Russischen, zum Englischen, zum Französischen, zum Dänischen, zum Norwegischen usw. - besonders unangebracht, denn dadurch sind (vor allem für Nichtmuttersprachler) diese Wörterbücher für bestimmte, den zeitlichen Aspekt betreffende lexikologische Untersuchungen - z.B. zu Ausbau und Differenzierung des Wortschatzes - wenig geeignet.

Die Einschränkungen, die soeben zum theoretisch-linguistischen und neographischen Forschungsstand vorgebracht werden mußten, stehen nicht im Widerspruch zu der Tatsache, daß in populären Beiträgen, Artikeln und Miszellen die Neologismen durchaus beträchtliche öffentliche Resonanz finden. Das bestätigt die Erfahrung, daß es sprachliche Gegenstände gibt, die für Nichtlinguisten von besonderem Interesse sind und hier - zum Teil heftig - diskutiert werden, während ihnen von sprachwissenschaftlicher Seite nur beschränkte Aufmerksamkeit geschenkt wird (z.B. auch orthographische Probleme oder die Fremdwortproblematik). Merkwürdig ist andererseits auch die Beobachtung,

11 Die Bibliographie "Deutsche Wörterbücher" von P. KƯHN (Tübingen 1978) kennt den Typ des Neologismenwörterbuches nicht. Aus neuerer zeit gibt es nur einige kleinere von HELLWIG, KINNE/STRUBE-EDELMANN, CONSTANTIN, HEBERTH . 
456

daß die in allgemeinen wörterbüchern so umsichtig markierten Archaismen nur selten zum Gegenstand lexikologisch-theoretischer Untersuchungen gemacht worden sind. 12

3.4. Wenden wir uns schließlich noch der Frage zu, wie die diachronische Markierung in mittleren allgemeinen Wörterbüchern am günstigsten aussehen sollte, d.h. welche Markierungsprädikate vorzusehen sind. Die Erwägungen darüber sind von unserem zentralen Anliegen bestimmt, dem Wörterbuchbenutzer die Dynamik im Wortschatz auch in einem synchronen Schnitt durch das Kontinuum ständiger sprachlicher Entwicklung und Veränderung $z u$ vermitteln und das Prozeßhafte - so gut es geht - auch im statischen Abbild zu zeigen.

Wir schlagen vor, mindestens folgende vier Stufen der Dimension "Zeit" anzunehmen:

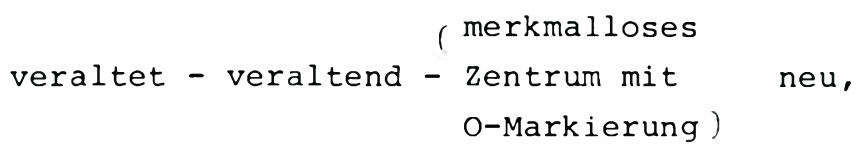

und also mit den Markierungsprädikaten veraltet, veraltend und neu $\mathrm{zu}$ arbeiten.

Die Verwendung zweier Prädikate - veraltend und veraltet - zur Markierung auf der Seite der Archaisierung, wie sie bereits von WDG, DUDEN-GWB und DUDEN-DUW praktiziert wird, stellt den Versuch dar, den Verlust an kommunikativer Relevanz als Proze $\beta$ zu erfassen. Wenn dieses Verfahren auch bestimmte Einordnungsprobleme mit sich bringt, wie z. B. SCHMIDT (1982) anhand eines Wörterbuchvergleiches nachgewiesen hat (vgl. auch HERBERG 1976), so kann seine Praktikabilität nicht grundsätzlich in Frage gestellt werden. Vielmehr sollte an der methodischen Verbesserung der zuordnungsentscheidungen (z.B. durch die Schaffung von Vergleichsmöglichkeiten innerhalb der wörterbuchkarthotek) gearbeitet werden.

Die Prozeßhaftigkeit i $\mathrm{n} n$ e $r \mathrm{~h}$ a $\mathrm{l}$ b der Neologie darstellen zu wollen, verbietet sich dagegen und ist u.w. auch noch nicht versucht worden. Geht man, wie es uns allein sinnvoll erscheint,

12 Ausdrücklich thematisiert werden sie in nur wenigen Beiträgen wie z.B. HERBERG (1976), SCHMIDT (1982). 
von einem Begriff des Neologismus aus, bei dem nicht nur sein Aufkommen und seine Verbreitung, sondern auch seine übernahme in den allgemeinen Gebrauch, also seine Approbation in der Kommunikationsgemeinschaft, in einem abzusteckenden zeitraum erfolgt sein müssen, so kommen als Kandidaten für die Aufnahme ins wörterbuch ohnehin nur solche lexikalischen Innovationen in Abgrenzung $\mathrm{zu}$ Okkasionalismen und Individualbildungen in Frage, bei denen der Prozeß der Eingliederung bereits zu einem gewissen Abschluß gekommen ist. Dennoch ist der mitunter daraus gezogene Schluß, daß man deshalb auf eine Markierung ganz verzichten könne, nicht logisch, da sie relativ zum Beginn des Erfassungszeitraumes des Wörterbuches das Merkmal "neu" tragen. Allerdings ist auf diesem Pol den Anforderungen der diachronischen Markierung mit einem, nämlich dem Prädikat neu Genüge getan; dieses sollte aber unverzichtbar sein. Eine Ermessensfrage dagegen ist es, ob man wie das WDG eine Differenzierung der Neologismen-Markierung nach "iewort, ifuprägung und Neubedeutungvornimmt, die nach unserer Kenntnis so hier erstmals angewandt worden ist. ${ }^{14}$ So dankbar zweifellos viele Benutzer für solche detaillierten Markierungen sind zumal für das Deutsche bisher ein Neologismen-Wörterbuch fehlt -, ist doch zu fragen, ob ein allgemeines wörterbuch damit nicht überfordert ist. Auch wenn nur 3,8\% des im WDG enthaltenen Wortschatzes derart als Neologismen ausgewiesen sind

13 Das schließt natürlich nicht aus, daß bei einem relativ großen Erfassungszeitraum, wie ihn das WGD hat, bestimmte Neologismen mit durchaus gesellschaftlicher Approbation innerhalb dieses Zeitraumes bereits wieder $z u$ veralten beginnen oder sogar schon veraltet sind, wofür diverse Neologismen aus der frühen Nachkriegszeit immer wieder als Beispiele genannt werden (vgl. HERBERG 1976, SCHMIDT 1982).

14 Über die Genese, die schließlich zu diesen Prädikaten geführt hat, geben einige der Beiträge in KLAPPENBACH (1980) Auskunft. 
(vgl. SPARMANN 1979, 104) 15 , so ist die 3467 malige Markierung mit solchen relativ umfangreichen Prädikaten schon als Druckraum-Problem nicht zu unterschätzen. Für eine so differenzierte Art der Markierung, die für ein Neologismenwörterbuch durchaus angebracht ist und dort womöglich noch zu verfeinern wäre, haben auch allgemeine Wörterbücher anderer Sprachen keine Notwendigkeit gesehen. (Im übrigen werden Hinweise auf die Art der Wortbildung oder der Ubernahme in den heimischen Wortschatz beim Hauptteil des Wortschatzes nicht gegeben.) Wenn wir an dieser Stelle abbrechen, so sind wir uns sehr wohl der Tatsache bewußt, daß zahlreiche der mit der diachronischen Markierungen in allgemeinen einsprachigen wörterbüchern des Deutschen verbundenen Probleme hier gar nicht zur sprache gekommen sind, so z.B. die Kombination dieser Markierungen mit Prädikaten anderer Markierungsdimensionen (besonders der Stil ebenen/Stilfärbungen) oder mit den Kennzeichnungen DDR bzw.BRD (vgl. dazu BRAUN 1981).

Das Anliegen dieses Beitrages soll abschließend thesenartig zusammengefaßt werden.

4. Zusammenfassung

(1) Im Netz diasystematischer Markierungen von Lexemen und Sememen in allgemein einsprachigen wörterbüchern wird die Markierungsdimension "Zeit" fast durchweg in irgendeiner Weise berücksichtigt. Zumeist erfolgt die Markierung durch die Verwendung von 1 - n diachronischen Markierungsprädikaten .

(2) Diachronische Markierungen haben primär die Aufgabe, den im Wörterbuch erfaßten Wortschatz unter dem Aspekt des Wandels im lexikalischen Teilsystem zu klassifizieren, wobei die Bearbeiter die Kommunikationsgemeinschaft zur

15 Der Anteil des als veraltet und veraltend markierten wortschatzes im WDG ist, wie HERBERG $(1976,2)$ an einer Stichprobe errechnet hat, mit $3,7 \%$ interessanterweise etwa ebensogroß. 
Zeit des synchronischen Schnitts in der Einheit ihrer Generationen zum Maßstab machen. Sekundär vermitteln diachronische Markierungen dem Benutzer Hinweise auf bestimmte Gebrauchsbedingungen (Restriktionen bzw. Präferenzen) sowie auf Vorkommenhäufigkeiten.

(3) Die diachronischen Markierungssysteme der Mehrzahl der deutschsprachigen und vieler anderssprachiger wörterbücher sind relativ zu ihrem primären (und damit auch zu ihrem sekundären) Zweck defektiv und haben dann nicht den Status einer Markierungsdimension sui generis.

(4) Der Defekt in der Markierung liegt auf der Seite des Innovativen (Neologismen), wohingegen das Veralten im lexikalischen System ziemlich regelmäßig markiert wird.

(5) Um einerseits den diachronischen Aspekt in der synchronischen Wortschatzdarstellung so weit wie möglich als Prozeß hervortreten zu lassen und andererseits die Möglichkeiten eines allgemeinen wörterbuches nicht zu überfordern, wird eine diachronische skala mit vier stufen und drei Markierungsprädikaten vorgeschlagen:

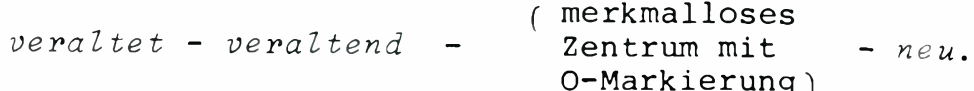




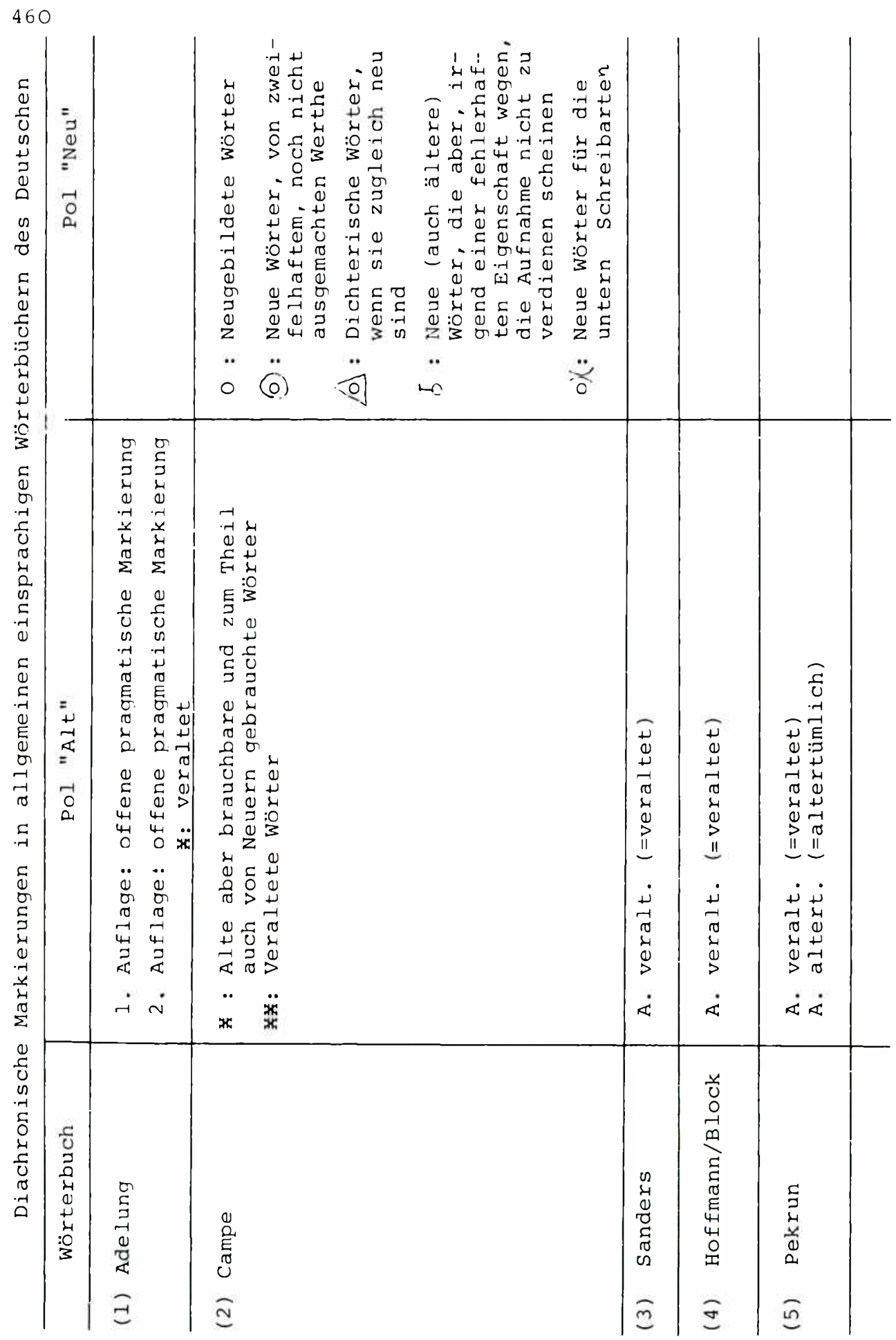




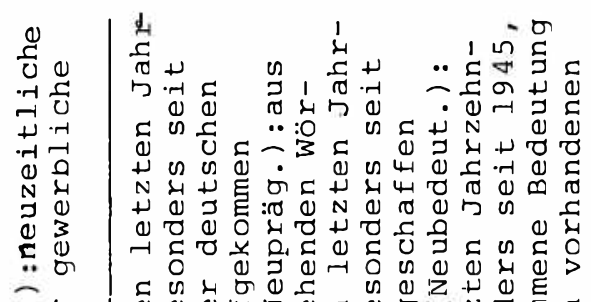

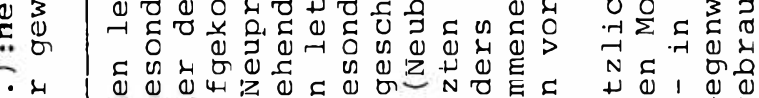

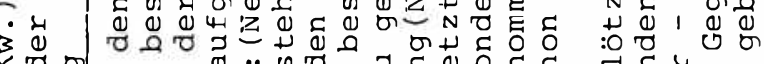
उ ப 东 ब 0 म

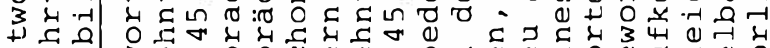

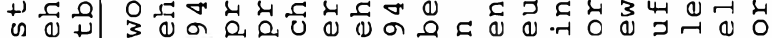
牙热

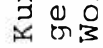
i ; z $z$

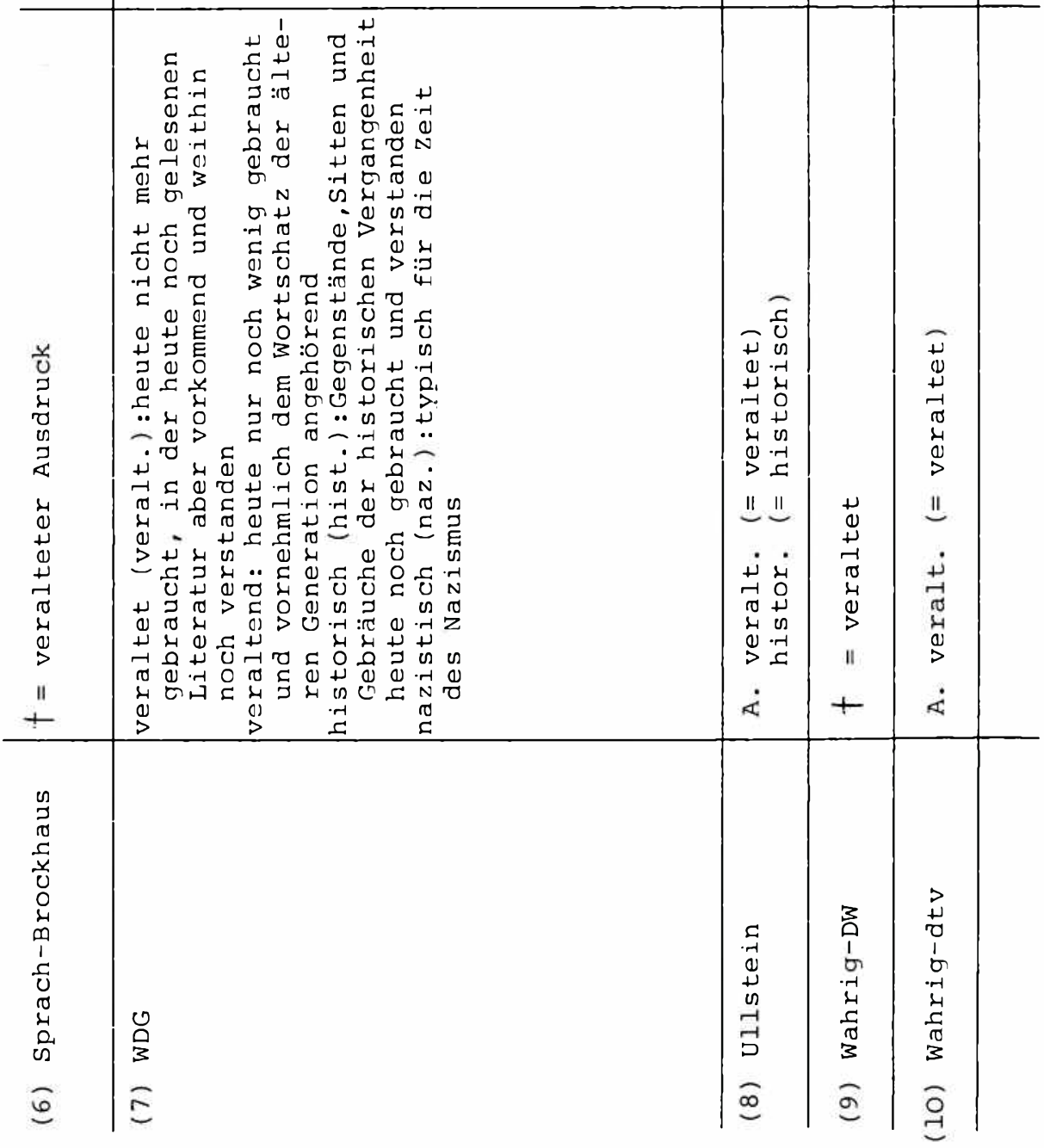




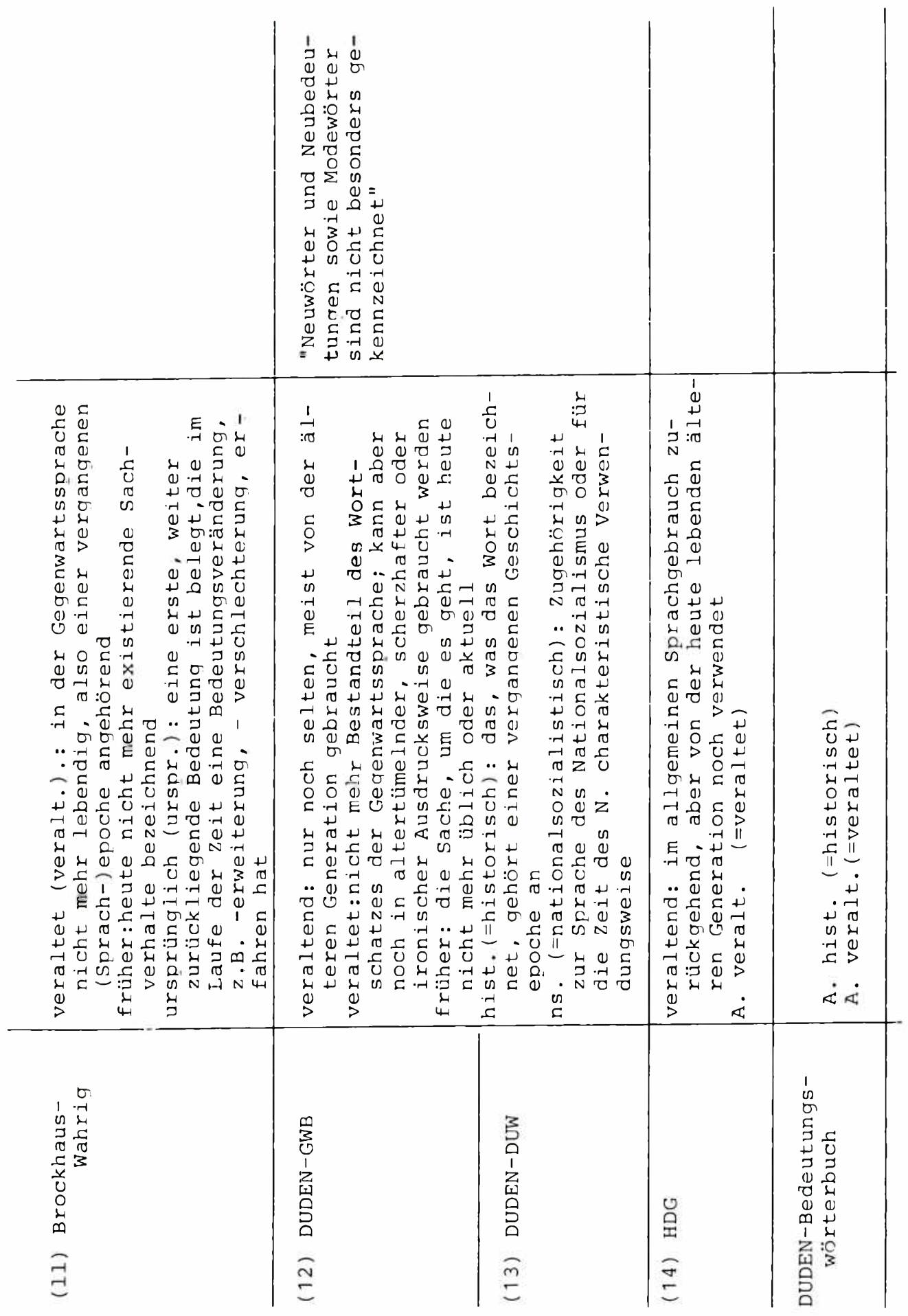




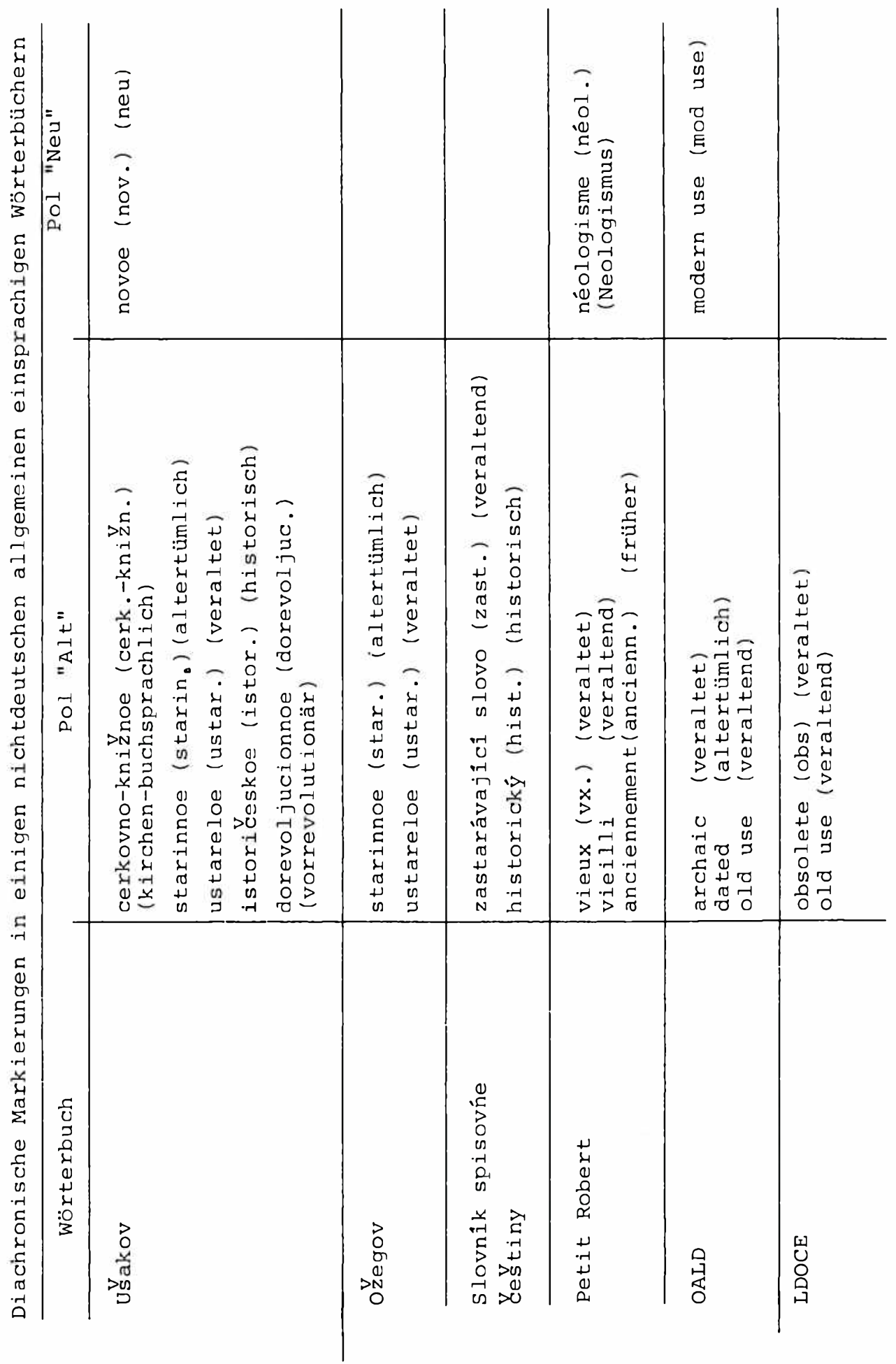




\section{LITERATUR}

I. Wörterbücher

ADELUNG = Johann Christoph Adelung: Versuch eines vollständigen grammatisch-kritischen Wörterbuches Der Hochdeutschen Mundart, mit beständiger Vergleichung der übrigen Mundarten, besonders aber der oberdeutschen. 5 Teile. Leipzig 1774-1786.

BROCKHAUS-WAHRIG = Brockhaus-Wahrig! Deutsches Wörterbuch in sechs Bänden. Hrsg. von Gerhard Wahrig ( $T$ ), Hildegard Krämer, Harald Zimmermann. Wiesbaden/Stuttgart 1980-1984.

CAMPE = Joachim Heinrich Campe: Wörterbuch der Deutschen Sprache. 5 Teile. Braunschweig 1807-1811.

CONSTANTIN = Theodor Constantin: Plaste und Elaste. Ein deutsch-deutsches Wörterbuch. Berlin 1982. DUDEN-Bedeutungswörterbuch = Duden. Bedeutungswörterbuch. 2., völlig neu bearbeitete und erweiterte Auflage. Hrsg. und bearb. von Wolfgang Müller unter Mitwirkung folgender Mitarbeiter der Dudenredaktion: Wolfgang Eckey (...) Mannheim/Wien/Zürich 1985 (Der Duden;Bd.10)

DUDEN-DUW = Duden. Deutsches Universalwörterbuch. Hrsg. und bearb. vom Wissenschaftlichen Rat und den Mitarbeitern der Dudenredaktion unter Leitung von Günther Drosdowski. Mannheim/Wien/Zürich 1983.

DUDEN-GWB = Duden. Das große Wörterbuch der deutschen Sprache in sechs Bänden. Hrsg, und bearb. vom Wissenschaftlichen Rat und den Mitarbeitern der Dudenredaktion unter Leitung von Günther Drosdowski. Mannheim/Wien/ Zürich 1976-1981.

HDG $=$ Handwörterbuch der deutschen Gegenwartssprache. In zwei Bänden. Von einem Autorenkollektiv unter der Leitung von Günter Kempcke. Berlin 1984.

HEBERTH = Alfred Heberth: Neue wörter. Neologismen in der deutschen Sprache seit 1945. Wien 1977.

HELLWIG = Gerhard Hellwig: Kennen Sie die neuesten Wörter? München ${ }^{3} 1983$.

HOFFMANN/BLOCK = P.F.L. Hoffmann: Wörterbuch der deutschen 
Sprache in ihrer heutigen Ausbildung. Bearb.

von Dr. Martin Block. Elfte, verbesserte Auflage.

Leipzig 1942 .

KINNE/STRUBE-EDELMANN = Michael Kinne/Birgit Strube-Edelmann:

Kleines Wörterbuch des DDR-Wortschatzes. Düsseldorf

1980 .

LDOCE = Longman Dictionary of Contemporary English.

Editor-in-Chief Paul Procter. 3. Aufl. 0.0. 1981.

OALD = Oxford Advanced Learner's Dictionary of Current English.

A S Hornby with A P Cowie, A C Gimson.

20th Impression (of 3rd Edition 1974). Oxford 1984.

OŽEGOV= Sergej IvanoviZ OŽegov: Slovar'russkogo Jazyka.

Izdanie 16-e, ispravlennoe. Pod redakciej (...)

N. Ju Svedovoj. Moskva 1984.

PEKRUN = Das Deutsche Wort. Nach den amtlichen Regeln bearbei-

tet von Richard Pekrun. Heidelberg 1953.

PETIT ROBERT = Le Petit Robert. Dictionnaire alphabétique

\& analogique de la langue francaise par Paul Robert.

Rédaction dirigée par A. Rey et J. Rey-Debove.

Paris 1979 .

SANDERS = Daniel Sanders. Wörterbuch der Deutschen Sprache.

2 Bde. in 3 Teilen. Leipziq 1860-1865.

slovnik spisovné Ceštiny = slovnik spisovné Cě̌tiny pro

ykolu a veřejnost. Praha 1978.

SPRACH-BROCKHAUS = Der Sprach-Brockhaus. Deutsches Bildwörter-

buch für jedermann. Siebente, durchgesehene Auflage.

Wiesbaden 1958.

ULLSTEIN = Ullstein Lexikon der deutschen Sprache. Hrsg. und

bearb. von Rudolf Köster unter Mitarbeit von Harald

Hahmann, Heribert Hartmann und Franz Mehling. Frank-

furt/Berlin 1969.

USAKOV = Tolkovyj slovar' russkogo jazyka pod redakciej

D.N. USakova. 4 Bde. Moskva 1935-1940.

WAHRIG-dtv = dtv-Wörterbuch der deutschen Sprache. Hrsg. von

Gerhard Wahrig in Zusammenarbeit mit zahlreichen Wissenschaftlern und anderen Fachleuten. München

1978. (=dtv 3136). 
WAHRIG-DW = Gerhard Wahrig: Deutsches Wörterbuch. Hrsg. in Zusammenarbeit mit zahlreichen Wissenschaftlern und anderen Fachleuten. Mit einem "Lexikon der deutschen Sprachlehre". Einmalige Sonderausgabe. Ungekürzt. Gütersloh 1968 .

WDG = Wörterbuch der deutschen Gegenwartssprache. Hrsg. von Ruth Klappenbach und Wolfgang Steinitz. 6 Bde. Berlin 1964-1977.

\section{Sonstige Literatur}

BRAUN (1981) = Peter Braun: Vergleichende Untersuchungen $\mathrm{zu}$ deutsch-deutschen Wörterbüchern. In: Muttersprache $91,157-168$.

HAUSMANN $(1977)=$ Franz Josef Hausmann: Einführung in die Benutzung der neufranzösischen wörterbücher. Tübingen 1977.

- $\quad(1983)=$ Franz Josef Hausmann: Wörterbücher in Frankreich und Deutschland. Ein Vergleich. In: Studien zur neuhochdeutschen Lexikographie III. Hrsg. von Herbert Ernst Wiegand. Hildesheim/Zürich/New York 1983 (Germanistische Linguistik 1-4/82), 119-155.

- $\quad(1985)$ = Franz Josef Hausmann: Lexikographie. In: Christoph Schwarze/Dieter Wunderlich (Hrsg.): Handbuch der Lexikologie. Königstein/Ts. 1985, 367-411.

HERBERG $(1976)$ = Dieter Herberg: Veraltendes und Veraltetes in unserem Wortschatz. Bemerkungen und Beispiele $z u$ einem beachtenswerten Aspekt des Verhältnisses von Sprach- und Gesellschaftsentwicklung. In:Sprachpflege $25,1-5$.

- $\quad(1986)$ = Dieter Herberg: Zur Einleitung des Handwörterbuchs der deutschen Gegenwartssprache (HDG). In: ZPSK 39, 195-205.

KEMPCKE $(1985)$ = Günter Kempcke: Die Erarbeitung des "Handwörterbuchs der deutschen Gegenwartssprache". In: Die Brüder Grimm. Erbe und Rezeption. Stockholmer Sym- 
posium 1984. Hrsg. von Astrid Stedje (Stockholmer Germanistische Forschungen 32), Stockholm 1985, 131-141.

KLAPPENBACH $(1980)=$ Ruth Klappenbach/Helene Malige Klappenbach: Studien zur modernen deutschen Lexikographie. Hrsg. von Werner Abraham unter Mitwirkung von Jan F. Brand. (LINGUISTIK AKTUELL 1). Amsterdam 1980. KÜHN/PÜSCHEL (1982) = Peter Kühn/Ulrich Püschel: "Der Duden reicht mir". Zum Gebrauch allgemeiner einsprachiger und spezieller Wörterbücher des Deutschen. In: Studien zur neuhochdeutschen Lexikographie II. Hrsg. von Herbert Ernst Wiegand. Hildesheim/New York 1982 (Germanistische Linguistik 3-6/80, 121-151. LUDWIG $(1986)$ = Klaus-Dieter Ludwig: Nicht-denotative Informationen lexikalischer Einheiten. In: Studien zu einem Komplexwörterbuch der Mikro-, Medio- und Makrostrukturen. Erscheint in: LS/ZISW/A, Berlin 1986.

MATTAUSCH (1979) = Josef Mattausch: Das Wörterbuch der deutschen Gegenwartssprache. Versuch einer Bilanz. In: Deutsch als Fremdsprache 16, 213-219.

ROSSIPAL (1973) = Hans Rossipal: Konnotationsbereiche, Stiloppositionen und die sogenannten "Sprachen" in der Sprache. Hildesheim 1973 (Germanistische Linguistik $4 / 73)$.

SCHAEDER (1983) = Burkhard Schaeder: Häufigkeiten und Häufigkeitsangaben in neuhochdeutschen Wörterbüchern. Zur Rolle von Frequenzuntersuchungen in der Lexikographie. In: Studien zur neuhochdeutschen Lexikographie III. Hrsg. von Herbert Ernst Wiegand. Hildesheim/Zürich/ New York 1983 (Germanistische Linguistik 1-4/82), 239-274.

SCHMIDT (1982) = Günter Dietrich Schmidt: Veraltetes Wortgut in der deutschen Sprache der DDR. In: Muttersprache $92,129-145$.

SCHUMANN (1985) = Hanna Brigitte Schumann: Gehoben - bildungssprachlich - prestigious - veraltend. Markierungsversuche. In: Beiträge $z u$ theoretischen und prakti- 
schen Problemen in der Lexikographie der deutschen Gegenwartssprache.

Berlin 1985 (LS/ZISW/A/122), 135-193.

SMELEVA $(1975 / 1982)=$ I. N. Smeleva: Einige Fragen der Stilistik in einem allgemeinen Wörterbuch der Hochsprache. Zit. nach: Werner Wolski (Hrsg.): Aspekte der sowjetrussischen Lexikographie. Ubersetzungen, Abstracts, bibliographische Angaben. Tübingen 1982, 201-220. SPARMANN $(1979)=$ Herbert Sparmann: Neues im deutschen Wortschatz unserer Gegenwart (26. Fortsetzung und SchluB). In: Sprachpflege 28, 103-105.

WIEGAND $(1981)$ = Herbert Ernst Wiegand: Pragmatische Informationen in neuhochdeutschen Wörterbüchern. Ein Beitrag zur praktischen Lexikologie. In: Studien zur neuhochdeutschen Lexikographie I. Hrsg. von Herbert Ernst Wiegand. Hildesheim/New York 1981 (Germanistische Linguistik 3-4/79), 139-271.

WIEGAND/KUČERA (1981) = Herbert Ernst Wiegand/Antonin Kucera: Brockhaus-Wahrig: Deutsches Wörterbuch auf dem Prüfstand der praktischen Lexikologie, I. Teil: 1. Band $(\mathrm{A}-\mathrm{BT}) ; 2$. Band $(\mathrm{BU}-\mathrm{FZ})$. In: Kopenhagener Beiträge zur germanistischen Linguistik 18, 94-217. 